\section{Avaliação da sensibilidade do questionário de triagem para dor orofacial e desordens temporomandibulares recomendado pela Academia Americana de Dor Orofacial}

\author{
Ana Paula S. Manfredi, \\ Ariovaldo A. Da Silva ${ }^{2}$, \\ Laércio L. Vendite ${ }^{3}$
}

Resumo

I

ntrodução: As Desordens Temporomandibulares têm interpretação muito ampla e descrevem uma população geral de pacientes sofrendo de disfunção dos músculos e articulações da mandíbula, usualmente dolorosa ${ }^{1}$. Além da queixa de dor nos maxilares, dores de ouvido, cabeça e face, os pacientes com essas desordens muitas vezes têm movimentos mandibulares limitados ou assimétricos, e os sons da Articulação Temporomandibulares (ATM) mais descritos são os estalos. Objetivo: Com o intuito de fazer uma análise qualitativa e quantitativa do uso de um instrumento de auxílio diagnóstico, foi aplicado o "Questionário para Triagem para Dor Orofacial e DTM", recomendado pela Academia Americana de Dor Orofacial (Anexo 1), ainda não testado no Brasil. A população alvo foi de pacientes com queixas de dor não-dental na região orofacial, cefaléia, otalgia e/ou nas ATM que procuraram o ambulatório médico e odontológico (CSS/CECOM) mantido pela Unicamp para o atendimento de sua comunidade interna. Forma de estudo: Prospectivo clínico randomizado. Método: O questionário foi aplicado em 46 pacientes ( 40 mulheres e 6 homens), e posteriormente foi realizado um exame clínico específico para diagnóstico das DTM. Resultados: A análise estatística revelou que este questionário apresenta uma sensibilidade de $85.37 \%$ e uma especificidade de $80 \%$ para pacientes portadores de desordens musculares da região orofacial (Kappa $=0,454)$ e uma baixa sensibilidade e especificidade para desordens intraarticulares (Kappa $=0,043$ ). Conclusão: O questionário é útil e viável para uma pré-triagem das chamadas DTM, principalmente para os distúrbios miogênicos, mas não deve ser o único recurso utilizado para diagnóstico.

\section{The sensibility appreciation of the questionnaire for selection of orofacial pain and temporomandibular disorders recommended by the American Academy of Orofacial Pain}

Palavras-chave: desordens das ATM's, dor orofacial, sintomas auditivos, questionário, cefaléia.

Key words: TMJ disorders, orofacial pain, ear symptoms, questionnaire, migraine.

Summary

\begin{abstract}
I
ntroduction: The Temporomandibular Joint Disorders (TMJ Disorders) has very ample interpretation and describes a population of patients suffering from muscles dysfunctions and the muscles and toggles of jaw usually painful ${ }^{1}$. Beyond the complaints of pain in the cheek, ear pain and migraines the patients with these clutters many times have limited or anti-symmetrical jaw movements, and the sounds of the TMJ most described as "clicks". Aim: With intention to make qualitative and quantitative analysis of the use and acuracity of an instrument of diagnostic aid, we used the "Questionnaire for Selection for Orofacial Pain and TMJ Disorders", recommended for the American Academy of Orofacial Pain (attachment 1), not yet tested in Brazil. The target population was made of patients with complaints of dental pain in the orofacial region, chronic headache, ear ache and daily pain in the preauricular area or in the TMJ, that made they seek for medical and dental attention at the CSS/CECOM, an ambulatory that the Unicamp keeps for the attendance of its internal community. Study design: Prospective clinical randomized. Method: The questionnaire was applied to 46 patients ( 40 women and 6 men), with later a specific clinical examination that resulted diagnosis of TMJ Disorders. Results: The statistical analysis disclosed that this questionnaire presents a sensitivity of $85,37 \%$ and a specificity of $80 \%$ for carrying patients of muscular symptoms of the orofacial region $($ Kappa $=0.454)$ and a low sensitivity and specificity for articulate symptoms $($ Kappa $=0.043)$. Conclusion: The questionnaire is useful and viable for a daily selection of TMJ Disorders, mainly for the myogenic riots, but shouldn't be use as the only tool for diagnosis.
\end{abstract}

\footnotetext{
Cirurgiã Dentista, aluna de Pós-Graduação do Departamento de Otorrinolaringologia -FCM - Unicamp ${ }^{2}$ Professor Doutor da Disciplina de Otorrinolaringologia - FCM - Unicamp

${ }^{3}$ Livre Docente em Biomatemática do Departamento de Matemática Aplicada - IMECC - Unicamp Trabalho realizado no Ambulatório de Odontologia do CSS/Cecom da Unicamp

Endereço para correspondência: C.D. Ana Paula Sereni Manfredi - Av. Andrade Neves 707, conj. 301 - 13013-161 - Campinas/ SP - manfredi@correionet.com.br Artigo recebido em 10 de maio de 2001. Artigo aceito em 20 de julho de 2001
} 


\section{INTRODUÇÃO}

As Desordens Temporomandibulares (DTM), também chamadas de distúrbios craniomandibulares, constituem um conjunto de doenças que afetam não somente a articulação temporomandibular (ATM), mas também áreas extrínsecas às articulações.?

As DTM foram identificadas como a principal causa de dor não-dental na região orofacial ${ }^{1,10}$. O sintoma doloroso em geral está localizado nos músculos da mastigação, área pré-auricular e/ou nas $\mathrm{ATM}^{1}$, sendo consideradas uma subclasse das desordens musculoesqueléticas ${ }^{16}$.

As DTM têm interpretação muito ampla e descrevem uma população geral de pacientes sofrendo de disfunção dos músculos e articulações da mandíbula, usualmente dolorosa. Este não é um grupo homogêneo de pacientes, pois muitas etiologias e mecanismos diferentes de dor são responsáveis por apresentações similares. É imperativo diagnosticar definitivamente o fator causal específico, de modo a tratar efetivamente o paciente.

As dores descritas como faciais, nos maxilares, dores pré-auriculares, dores de ouvido, dores de cabeça, são comumente exacerbadas pela função da mandíbula e são conhecidas como distúrbios extra-capsulares. Desvios nos movimentos mandibulares, movimentos mandibulares limitados ou assimétricos podem ser resultado de hiperatividade dos músculos responsáveis pelo fechamento da mandíbula, mas também podem ser resultado de "desordens intra-capsulares"10. Nestes casos, os sons da ATM como cliques e/ou creptação, mais descritos como estalos, são os sinais mais freqüentes.

O sinal mais freqüente dos distúrbios extra-capsulares ou miogênicos é a dor facial difusa. Historicamente, o trabalho muscular excessivo foi considerado como causador de fadiga, que então levava à dor. Estudos mostraram que em indivíduos normais (não pacientes), ao se apertar os dentes voluntariamente ${ }^{5,6}$ ou forçar a protrusão ${ }^{18}$ mandibular, apresentavam dor aguda. Além disso, Christensen ${ }^{4}$ relatou que o ranger de dentes, os movimentos de lateralidade da mandíbula envolviam alongamento dos músculos masséter e pterigóideo medial, enquanto estes estavam se contraindo. Os sinais produzidos quando o músculo masséter é o maior envolvido são descritos como "dor na mandíbula". Quando há maior envolvimento do músculo temporal há a queixa de cefaléia. Os sinais de otalgia e dor retrobulbar indicam uma maior sensibilidade no músculo pterigóideo lateral. Dores à deglutição no ângulo da mandíbula, bem com odinofagia, são provenientes do pterigóideo medial.

Os distúrbios internos ou intra-capsulares são todos os processos anormais que ocorrem nos limites das ATM, incluindo não somente os deslocamentos discais, mas também a osteoartrose, artrite inflamatória, deformidades congênitas e anormalidades traumáticas, neoplásicas e do desenvolvimento. ${ }^{13}$
Avaliar estes pacientes requer atenta análise multidiscipinar. Perceber na anamnese a possibilidade de DTM direciona o restante do procedimento. O uso correto da abordagem clínica do paciente com dor facial permite o encaminhamento terapêutico adequado. Tendo observado que médicos e dentistas têm dificuldade em encaminhar corretamente os casos de dores orofaciais, o uso de anamnese dirigida pode facilitar este trabalho, orientando a pesquisa clínica e apressando o diagnóstico e a terapêutica.

\section{OBJETIVO}

O objetivo principal deste trabalho foi avaliar o grau de sensibilidade e especificidade do Questionário para Triagem para Dor Orofacial e DTM, recomendado pela American Academy of Orofacial Pain (Anexo 1) ainda não testado no Brasil e correlacionar esses achados com o exame clínico odontológico específico para diagnóstico de DTM.

\section{CASUÍSTICA E MÉTODO}

Para a análise a ser feita foi colhida uma amostra entre os pacientes atendidos no Ambulatório Médico e Odontológico (CSS/CECOM) existente dentro da Universidade Estadual de Campinas para atendimento da sua comunidade interna.

Foram analisados um total de 46 pacientes ( 40 mulheres e 6 homens), com uma idade média de 31 anos, que procuraram espontaneamente o atendimento junto ao ambulatório no período de janeiro a agosto de 2000 . Estes pacientes já haviam sido avaliados previamente por outros profissionais de saúde, e mantinham queixa de cefaléias, dores na região orofacial, estalos ou creptações na região auricular e pré-auricular e não apresentavam subsídios clínicos para enxaqueca, otopatias ou qualquer processo inflamatório ou infeccioso de origem dental.

Inicialmente, esses pacientes foram submetidos a uma avaliação médica, sendo constatadas que as queixas de cefaléia, dor auricular, pré-auricular e dor orofacial não eram positivos a sinais neurológicos ou otorrinolaringológicos; encaminhou-se estes pacientes a uma análise odontológica.

Neste ambulatório, os pacientes foram submetidos ao preenchimento do questionário auto explicativo para triagem recomendado pela American Academy of Orofacial Pain para Dor Orofacial e DTM (Anexo 1). O questionário é composto de dez perguntas direcionadas, com respostas sim/não, a sinais e sintomas mais freqüentes de dor orofacial e DTM (Gráfico 1).

Um exame clínico acurado, um exame pantorradiográfico e o termo de consentimento informado do Comitê de Ética em Pesquisa da Faculdade de Ciências Médicas da Universidade Estadual de Campinas. 
Anexo 1. Questionário auto-explicativo de Triagem.

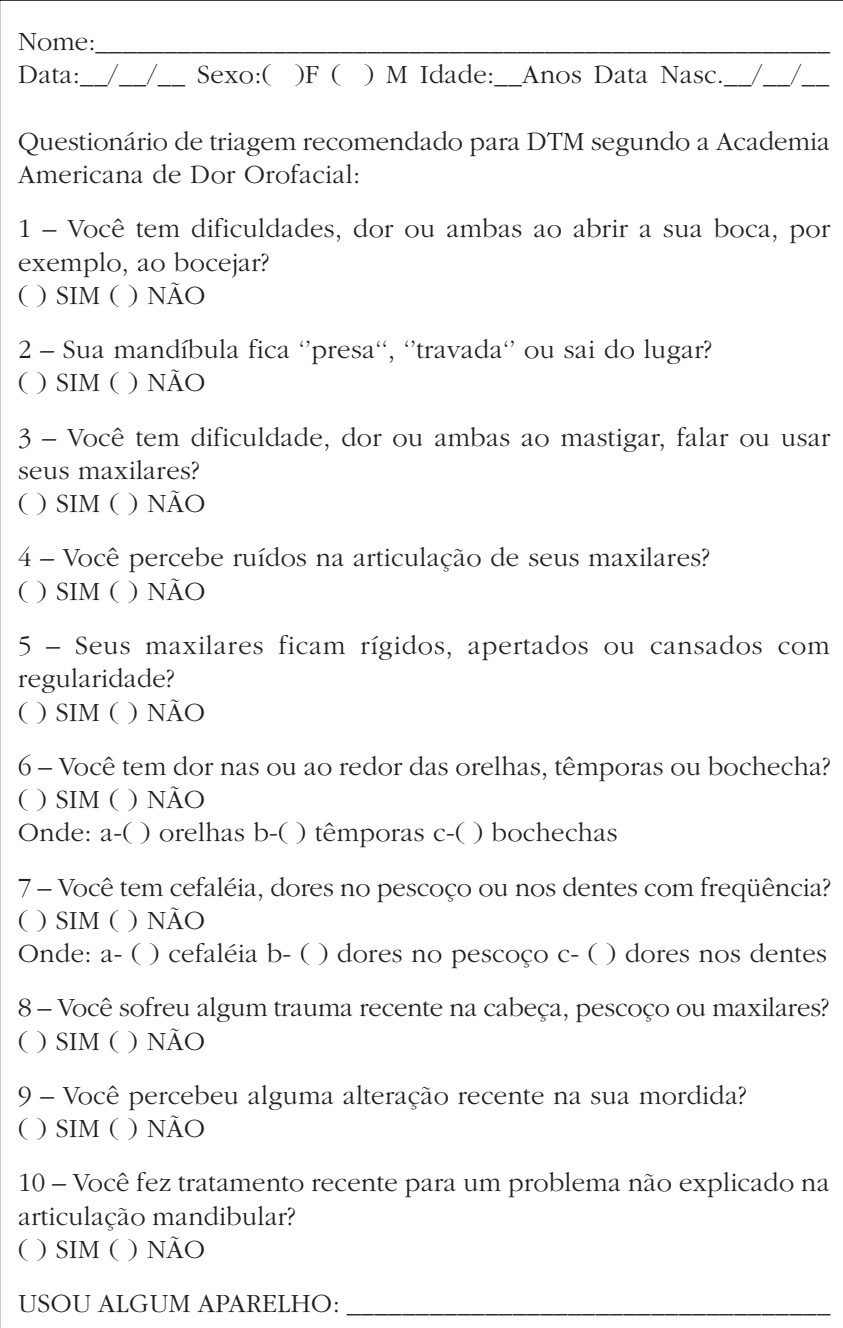

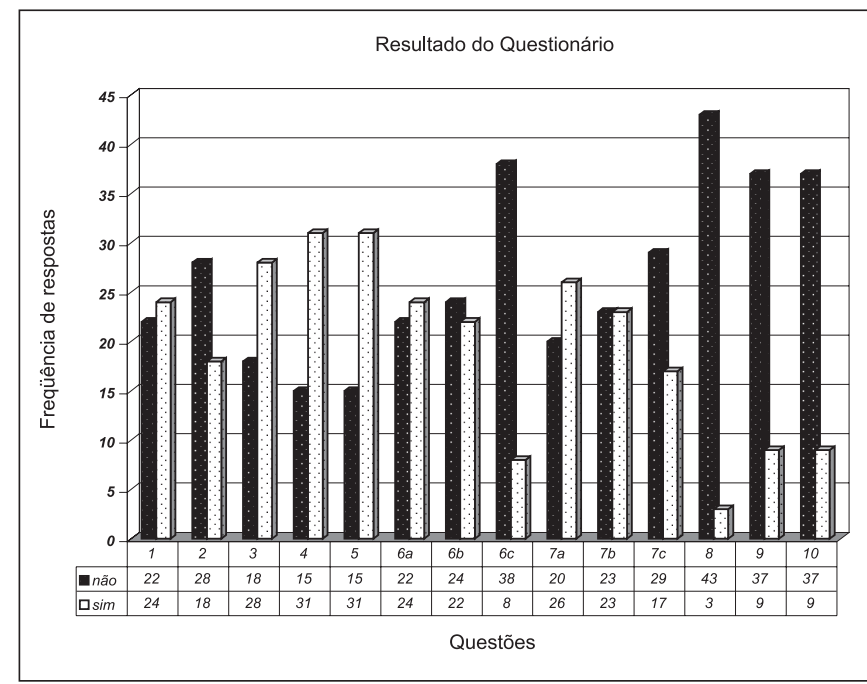

Gráfico 1. Sinais e sintomas mais freqüentes de dor orofacial e DTM.
No exame clínico odontológico foram avaliados os seguintes itens:

- Queixa principal;

- Presença de doença sistêmica (nenhum paciente portador de qualquer tipo de patologia sistêmica foi incluído neste trabalho);

- Máxima abertura bucal, sendo considerado o padrão normal de 40 a $45 \mathrm{~mm}$;

- Estalido das ATM ao abrir e fechar a boca, através de palpação digital bilateral;

- Presença de desgaste dental, indicativo de uma possível parafunção como o ranger de dentes;

- Relato direcionado para presença de um quadro de stress do paciente;

- Histórico de macrotrauma na região orofacial, principalmente "wiplash";

- Tratamento ortodôntico prévio;

- Palpação da musculatura mastigatória e região retrocondilar.

A avaliação da condição dolorosa muscular mediante palpação foi realizada por somente um avaliador durante a primeira consulta, e a classificação da intensidade da dor foi definida através do seguinte escore:

$0 \rightarrow$ nenhuma dor;

$1 \rightarrow$ dor fraca;

$2 \rightarrow$ dor média;

$3 \rightarrow$ dor forte.

Os músculos avaliados foram: pterigóideo medial, masséter, temporal (elevadores da mandíbula), milohioideo e esternocleidomastoídeo, bem como a região retrocondilar com o paciente de boca aberta. Também foram avaliados traumas dentais, a oclusão dental, tratamento ortodônticos prévios e traumas locais e cervicais.

A pantorradiografia ou radiografia panorâmica foi pedida para o auxílio diagnóstico de um eventual quadro infeccioso crônico ou subagudo de origem dental, bem com para afastar a hipótese da presença de cistos ou neoplasias.

\section{RESULTADOS}

A análise descritiva através de tabelas de freqüencia e medidas de posição e dispersão do questionário e avaliação clínica demonstrou que:

As questões 1 e 2 do questionário relacionadas ao movimento de abertura e fechamento bucal: como a percepção do paciente à normalidade deste movimento varia de um indivíduo para outro, este item foi verificado na avaliação clínica e, muito embora tenha havido $52,2 \%$ e $39,1 \%$ de respostas positivas nas questões 1 e 2 , respectivamente, a média de máxima abertura bucal dos pacientes foi de $49 \mathrm{~mm}$.

As questões 3 e 5 são direcionadas aos sinais dolorosos característicos de DTM como dificuldade e/ou 
dor à mastigação e fala, bem como a sensação de cansaço nos maxilares ${ }^{5,6}$, fazendo destas duas perguntas as mais significativas do questionário. Estes sinais estão relacionados ao uso inadequado do aparelho estomatognático que pode ser devido à condição oclusal do paciente, bem como à presença de hábitos de apertar e/ou ranger os dentes. Por este motivo, o percentual de respostas positivas foi de $60,9 \%$ e $67,4 \%$, respectivamente e a correlação com a avaliação clínica foi significativa (Kappa=0.449).

A questão 4 que relata a presença de ruídos nas $\mathrm{ATM}^{7}$ teve $67,4 \%$ de respostas positivas e apenas $30,4 \%$ dos sujeitos tiveram confirmada a presença de estalido intra-capsular na avaliação clínica.

As questões 6 e 7 listam os principais sintomas de dor orofacial e distúrbios extra-capsulares das ATM, que são: dor ao redor das orelhas ( $47,8 \%$ respostas negativas e $52,2 \%$ respostas positivas), têmporas $(52,2 \%$ respostas negativas e $47,8 \%$ respostas positivas), ou bochechas (82,6\% respostas negativas e $17,4 \%$ respostas positivas); cefaléias (43,5\% respostas negativas e 56,5\% respostas positivas); dores no pescoço (50,0\% respostas negativas e $50,0 \%$ respostas positivas), e nos dentes (63,0\% respostas negativas e $37,0 \%$ respostas positivas).

A questão 8 tem como objetivo principal investigar a presença de macro-trauma, como quedas, acidentes automobilísticos, traumas diretos na maxila ou na mandíbula ou na região craniocervical ${ }^{16}$, visando relacionálos aos sinais e sintomas da queixa principal do paciente. Nesta amostra o percentual de pacientes acometidos de algum trauma recente na cabeça foi de apenas 6,5\%.

$\mathrm{Na}$ questão 9, a percepção pelo paciente de sua condição dental é relevante pois é um indicativo que os sinais da queixa principal podem estar relacionado com o sistema mastigatório e o percentual de respostas positivas foi de $19,6 \%$ e negativas de $80,4 \%$ positivas.

A questão 10 informa se o paciente já fez ou está fazendo algum tratamento odontológico para algum problema nas ATM.

$\mathrm{Na}$ avaliação clínica, os itens responsáveis pelo desgaste dental, resultado de uma provável parafunção, como o bruxismo, e o stress, foram os possíveis fatores etiológicos mais significativamente encontrados no exame clínico (Gráfico 2).

Os músculos que apresentaram a maior freqüência de dor nos escores 2 (dor média) e 3 (dor forte) e o maior percentual de pacientes afetados foram o pterigóideo medial e o esternocleidomastoídeo (Gráfico 3 e 4).

\section{DISCUSSÃO}

A avaliação clínica direcionada e específica para DTM ressaltou alguns possíveis fatores etiológicos mais incidentes nesta amostra, e dentre estes o desgaste dental. A oclusão dental ainda é considerada um dos fatores

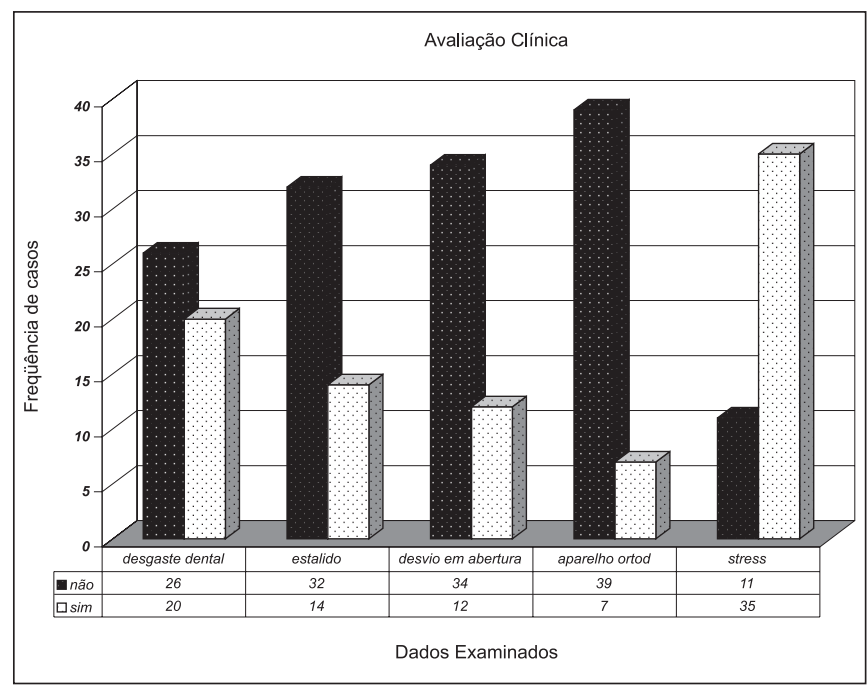

Gráfico 2. Achados de exame clínico.

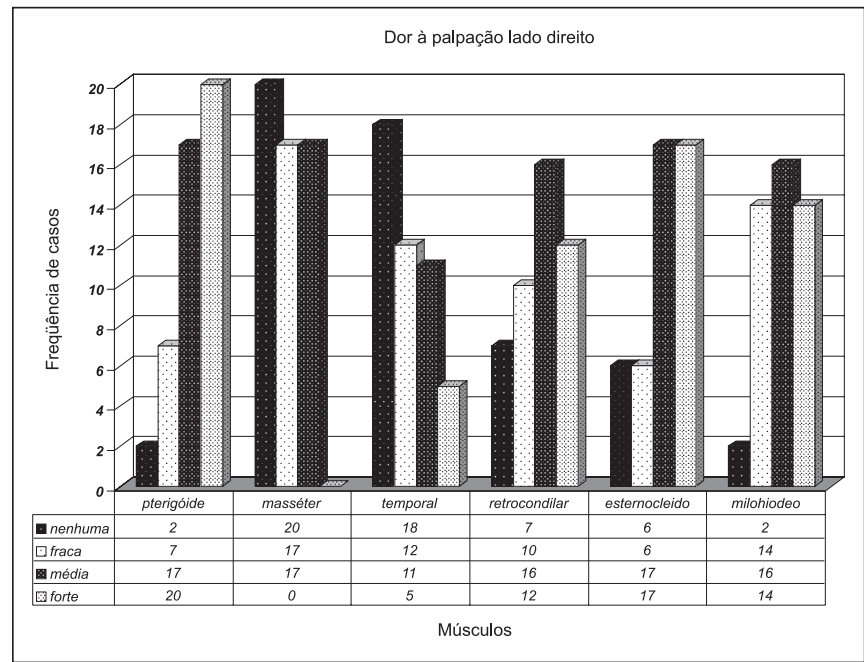

Gráfico 3. Freqüência de dor à palpação de musculatura do lado direito.

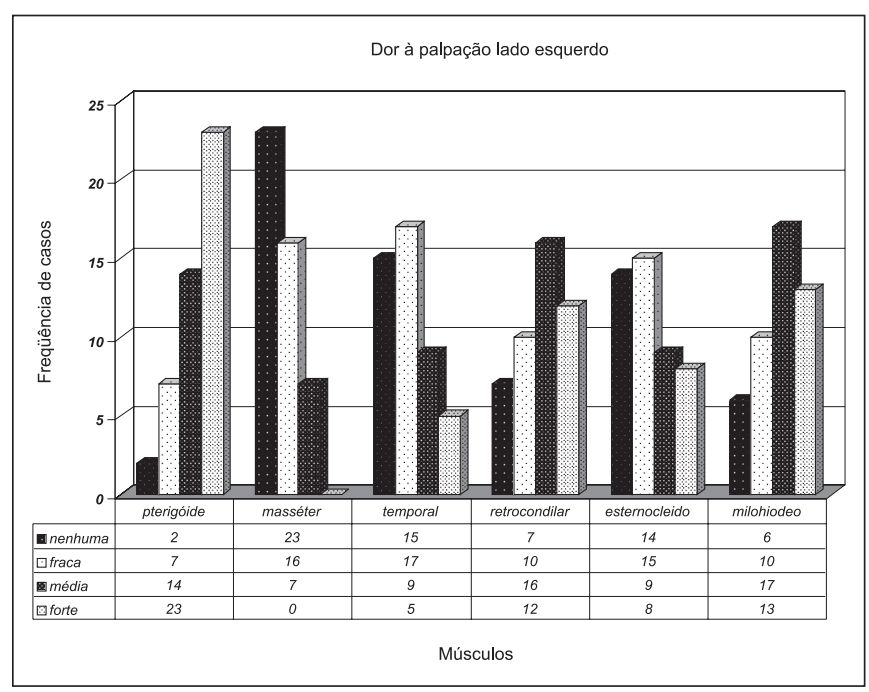

Gráfico 4. Freqüência de dor à palpação de musculatura do lado esquerdo. 
predisponentes ao aparecimento das desordens temporomandibulares ${ }^{6}$, já que um encaixe dental instável pode levar a um desequilíbrio da musculatura mastigatória, sendo sobrecarregado o lado de preferência da mastigação. Quando o paciente é portador de alguma prótese bucal, desgastes nos dentes artificiais podem ocasionar diminuição da altura normal do terço inferior da face, causando danos às ATM. Esta relação oclusão $\Leftrightarrow$ desordem temporomandibular já foi muito maior ${ }^{11}$, e o tratamento ortodôntico era a única forma de tratamento para as dores orofaciais, mas estudos recentes mostraram que, ocasionalmente, após mudanças na oclusão dental, os pacientes desenvolviam bruxismo e dor em conseqüência destas mudanças ${ }^{16}$.

Na palpação muscular verificou-se que o músculo esternocleidomastoídeo apresentou-se sensível à palpação com dor nos escores 2 (dor média) e 3 (dor forte) em $41,3 \%$ e $21,7 \%$ respectivamente. O músculo esternocleidomastó́deo, que possui sua inervação sensitiva proveniente do segundo e terceiro nervos cervicais, não é um músculo diretamente relacionado à mastigação, e o resultado à palpação dolorosa ser significativa deve-se a impulsos nervosos do $\mathrm{V}$ par (nervo Trigêmeo), provenientes do tecido periodontal, lingual, das ATM e de receptores musculares, que podem influenciar ramos de motoneurônios deste músculo. Impulsos trigeminais devem ser considerados, devido à relação existente entre tratos descendentes do nervo trigeminal e raízes cervicais altas. Neurônios das três divisões do nervo trigêmeo e dos nervos cranianos do VII, IX, e X pares dividem os mesmos feixes neuronais com neurônios provenientes dos primeiros segmentos cervicais ${ }^{18}$.

Para verificar a concordância entre a avaliação do questionário e a avaliação clínica foi utilizado o coeficiente $\mathrm{Kappa}^{8,9}$. A interpretação da magnitude deste coeficiente é definida como: valores maiores ou iguais a 0.75 indicam excelente concordância, valores entre 0.75 e 0.40 indicam boa concordância e valores menores ou iguais a 0.40 não indicam concordância.

A análise estatística avaliou as respostas positivas e negativas encontradas no questionário e correlacionou com achados clínicos da anamnese específica para DTM. Foram feitas várias correlações entre as questões mais indicativas de uma possível patologia temporomandibular. Os dados encontrados foram:

1. Questão 1 ou 2 positiva e máxima abertura bucal abaixo de $40 \mathrm{~mm} \rightarrow$ Kappa $=0.090$. A dificuldade do movimento de abertura bucal poderia dar margem para interpretação da presença de um distúrbio intra-capsular (Incoordenação Côndilo-disco sem redução, ou "closed lock", onde o disco capsular se posiciona à frente do côndilo mandibular e não é recuperado durante a abertura bucal) $^{7}$. Porém não foi verificado em nenhum dos sujeitos da amostra. Este achado vem corroborar um modelo proposto por Lund e colaboradores, onde a dor provoca a limitação dos movimentos mandibulares de duas maneiras:

- pela restrição voluntária do movimento até um grau livre de dor;

- pela ativação reflexa dos músculos antagonistas durante um movimento, em que a velocidade e amplitude do movimento sejam ambas reduzidas.

2. Questão 3 positiva com escores 2 ou 3 para dor no músculo pterigoídeo medial $\rightarrow$ Kappa $=0.449$, porém outros músculos correlacionados isoladamente com questões específicas, como as questões 6 e 7, não apresentaram uma correlação considerada significativa. Optou-se então para uma associação de questões como as Questões 3 e 5 com o conjunto de músculos elevadores da mandíbula ( pterigoídeo medial, masséter e temporal) e o resultado foi significativo, Kappa $=0.454$, e a sensibilidade do questionário para este tipo de avaliação dolorosa foi de $85.37 \%$ e a especificidade de $80.00 \%$.

3. Questão 4 positiva e presença de estalido nas ATM ao abrir e fechar a boca $\rightarrow$ Kappa $=0.043$. Este achado é de grande valia, pois 31 pacientes afirmaram apresentar estalidos nas ATM e tal fato não foi confirmado pelo exame clínico. O estalido intra-capsular, ou incoordenação côndilo-disco com redução, ocorre quando o disco capsular se encontra posicionado erroneamente à frente do côndilo mandibular, sendo resgatado ao se abrir a boca com recuperação da posição correta ${ }^{12}$. Um estudo eletromiográfico e artrográfico realizado em pacientes com estalidos à função mandibular e pacientes normais, de Zijun; Huiyon \& Weiya (1989) ${ }^{14}$, concluiu que a hiperfunção dos músculos pterigoídeos medial e lateral, em pacientes com artrografia normal, podem produzir sons nos movimentos mandibulares, que são diferentes dos estalidos característicos de desordens intracapsulares, embora este mecanismo de hiperfunção muscular possa até contribuir para o desenvolvimento de tal patologia.

\section{CONCLUSÕES}

- O questionário é sensível e correlato para patologias extra-capsulares ou distúrbios miogênicos onde a queixa principal é a dor facial difusa e irradiada.

- Não define presença e gravidade de patologias intracapsulares.

- Indica a necessidade de avaliação multidisciplinar para pacientes com queixas de dores de cabeça, dores na face, dores na região auricular, pré-auricular e estalidos nas articulações. 


\section{REFERÊNCIAS BIBLIOGRÁFICAS}

1. BELL, W. E. - Tempromandibular Disorders. Classification, Diagnosis, Management. $3^{\text {rd }}$. Chicago: Year Book, 166-76, 1990.

2. CLARK, G.T.; DECANHO, R.E.; GOULET, J.P. - The utility and validity of current diagnosis procedures for defining temporomandibular joint disorders patients. Adv Dent Res; 7(2):97-112, 1993.

3. CLARK, G.T.; SELIGMAN, D.A.; SOLBERG, W.K. ET AL. - Guidelines for the examination and diagnosis of temporomandibular Disorders. J Am Dent Assoc, 106:75-8, 1983.

4. CHRISTENSEN, L.V. - Pain from the jaw muscles in children and adults. In: GRABER, L.W., ed. Orthodontics, state of art; essence of the science. St. Louis: Mosby: pp.28-47, 1986.

5. CLARK, G.T.; JOU, R.W.; LEE, J.J. - Jaw pain and stiffness levels after repeated maximum voluntary clenching. J Dent Re, 68:69-71, 1989.

6. CHRISTENSEN, L.V.; MOHAMED, S.E.; RUGH, J.D. - Isometric endurance of human masséter muscle during consecutive bouts of tooth's clenching. J oral Rehabil, 12:509-14, 1985.

7. DOLWICK, M.F. - Intra-articular disc displacement: part1. It's questionable role in temporomandibular joint pathology. J Oral Maxillofac Surg 53:1069-72, 1995.

8. FLEISS, J.L. (1981). - Statistical Methods for Rates and Proportions. $2^{\text {a }}$ ed. John Wiley \& Sons Inc., Nova Iorque.

9. FLETCHER, R.; FLETCHER, S.; WAGNER, E.H. (1991). - Epidemiologia Clínica. $2^{\underline{a}}$ ed. Artes Médicas. Porto Alegre.
10. GRIFFITHS, R.H. - Report of President's Conference on Examination, Diagnosis and Management of Temporomandibular Disorders. J Am Dent Assoc. 106:75-7, 1983.

11. GRIFFITHS, R.H. - Report of President's Conference on Examination, Diagnosis and Management of Temporomandibular Disorders. J Am Dent Assoc, 106:75-8, 1983.

12. LOBBEZOO-SCHOLTE, A.M.; DE LEEW, J.R.J.; STEENKS, M. ET AL. Diagnostic subgroups of craniomandibular disorders. Part I: Self-report data and clinical findings. J Orofacial Pain, 9:24-36, 1995.

13. LASKIN, D.M.; GREENE, C.S. - Technological methods are the diagnosis and treatment of temporomandibular disorders. Quintessence Int 23:95, 1992.

14. LIU, ZIJUN; WANG, HUIYUN; AND, PUWEIYA. - A comparative eletromyographic study of the lateral pterygoid muscle and arthrography in patients with temporomandibular joint disturbance syndrome sound. J of Prosth Dent, 62:229-33, 1989.

15. NASSIF, J.; HILSEN, K. - Screening for temporomandibular disorders: History and clinical examination. J Prosthodont, 1:42-6, 1992.

16. OKESON, J.P. - Bell's Orofacial Pains. $5^{\text {th }}$ ed. Chicago: Quintessence: 123-33, 1995

17. SMITH, V.; WILLIAMS, B.; SAPLEFORD, R. - Rigid internal fixation and the effects on the temporomandibular joint and masticatory system: A prospective study. Am J Orthod Dentofac Orthop, 102:491-500, 1992.

18. SCOTT, D.S.; LUNDEEN, T.F. - Myofascial pain involving the masticatory muscles: an experimental model. Pain, 8:207-15, 1980. 